SCIDic

\section{Comparative Evaluation Of The Apical Sealing Ability Of BioRoot And Ahplus Sealers: An In Vitro Study}

Research Article

Ammar EID ${ }^{1}$, Davide Mancino², Fadi Joudi ${ }^{3}$, Mohammad Salem Rekab ${ }^{1}$, Kinda layous ${ }^{1}$, Omar HAMADAH4, Youssef Haikel², Naji KHAROUF²,

${ }^{1}$ Damascus University, Faculty of Dental Medicine, Department of Endodontics, Damascus, Syria.

${ }^{2}$ Department of Endodontics, Faculty of Dental Medicine, Strasbourg University, Strasbourg, France.

${ }^{3}$ Kalamoon University, Faculty of Dental Medicine, Department of Endodontics, Damascus, Syria.

${ }^{4}$ Department of Oral Medicine, Faculty of Dental Medicine, Damascus University, Damascus, Syria.

\title{
Abstract
}

Introduction: The aim of this study was to evaluate the ability of BioRoot sealer in preventing the dye penetration and to compare its filing ability with AH Plus sealer.

Materials and methods: 50mandibular premolars recently extracted, single-rooted, were selected. The samples were randomly divided in 2 equal groups $(n=25)$ for obturation: BioRoot sealer (Group 1) and AH Plus sealer (Group 2). Fifteen teeth of each group were prepared formicroleakage evaluation. The teeth were cleared using teeth clearing technique, then the teeth were examined by a stereo microscope (20x magnification) for evaluation of the linear dye penetration at the toothmaterials interface. The other 10 teeth of each group were prepared to evaluate the void percentages at $2 \mathrm{~mm}$ from the apex; andin order to observe and analyze the presence of intra-tubule tags. Data were statistically compared by One-way Analysis of Variance test.

Results: The results of the statistical analysis showed that there is a significant difference in apicalleakage between groups. The dye penetration in G2 $(0.76 \pm 0.66) \mathrm{mm}$ was more than in $\mathrm{G} 1(0.26 \pm 0.06) \mathrm{mm}$, suggesting that newly introduced BioRoot sealed the root canal better compared to AH Plus Sealer. No statistical significant difference was found between both sealers concerning the void percentages. SEM analyses showed the absence of intra-tubule tags for the two techniques analyzed at 2 $\mathrm{mm}$ from the apex.

Conclusion: A new calium cilicate-based root canal filling material (BioRoot) showed a good apical sealing-ability when it was used in a single cone technique comparing to AH Plus sealer in the mandibular premolar with one canal.

Keywords: AHPlus; BioRoot; Microleakage; Sealing Ability; SEM.

\section{Introduction}

The main objective of endodontic procedures is to offer threedimensional filling of the root canals and all accessory canals, that may prevents any connection between the periodental tissue and the root canal [1].

Among various types of sealer used today AH Plus (Dentsplysirona, De Trey Konstanz, Germany) has gained popularity due to its radiopacity, biocompatibility, ease to use and availability [2]. AH Plus is a hydrophobic epoxy resin-based sealer that has been used as the gold standard material in many research, It is a twocomponent root canal sealer, with a components epoxy resins, zirconium oxide, iron oxide, calcium tungstate and silicone oil [2-4]. Since it contains resin and has faster setting time, $\mathrm{AH}$ Plus tends to shrink and cause early debonding from the root canal wall [5].

BioRoot $^{\mathrm{TM}}$ RCS (Septodont, Saint-Maur-des Fosses, Franceis the newest endodontic sealer based on tricalcium silicate materials benefiting from both Active Biosilicate Technology and Biodentine $^{\mathrm{TM}}[6]$.

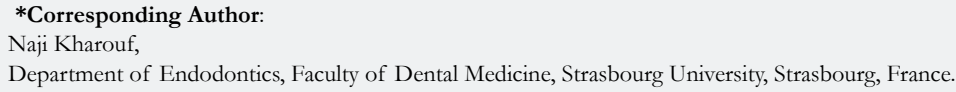

Citation: Ammar EID, Davide Mancino, Fadi Joudi, Mohammad Salem Rekab, Kinda Layous, Omar HAMADAH, Youssef Haikel, Naji KHAROUF. Comparative Evaluation Of The Apical Sealing Ability Of BioRoot And Ahplus Sealers: An In Vitro Study. Int J Dentistry Oral Sci. 2021;08(04):2309-2313. doi: http://dx.doi.org/10.19070/2377-8075-21000456 
BioRoot is a powder/liquid hydraulic tricalcium silicate-based cement (Gilles \& Oliver 2012) marketed since February 2015 and recommended for single cone technique or cold lateral condensation root filling. The powder contains tricalcium silicate, povidone and zirconium oxide; the liquid is an aqueous solution of calcium chloride and polycarboxylate $[7,8]$. BioRoot has been reported to induce in vitro the production of angiogenic and osteogenic growth factors by human periodontal ligament cells [9]. Moreover, it has a lower cytotoxicity than other conventional root canal sealers with hard tissue deposition [10]. Its antimicrobial activity was also reported [11].

Evaluation of sealing ability has been considered an important parameter to assess with the introduction of each new sealer. Dye penetration methods is a widely used methods [12-14]. Accordingly, several studies have used methylene blue as a dye because it is inexpensive and easy to perform, and easy to manipulate. It offers a high degree of staining and it has a molecular weight even lower than bacterial toxins [15].

The adaptation of a sealant to the dentin has generally been evaluated using stereo-microscopy, confocal laser microscopy, scanning electron microscopy (SEM), leakage tests, and digital imaging $[16,17]$.

The purpose of this in vitro study was to analyze microscopically (SEM, digital microscope and stereomicroscope) the ability of BioRoot sealer in preventing the dye penetration, and to compare its filing ability with AH Plus sealer. Further more, the voids at the interface between these sealers and dentin was apically evaluated.

The null hypothesis of this study was that there would be no difference between the two different materials of obturation in preventing the dye penetration and its filing ability.

\section{Materials and Methods}

\section{Sample Preparations}

Fifty extracted human permanent mandibular premolar single root teeth, free-caries, with fully formed apices were selected for this in vitro study. The ethics committee of Damascus University approved the protocol (protocol no. 1613/S). After removal of the external debris, teeth were placed in $1 \%$ sodium hypochlorite solution for $15 \mathrm{~min}$ and stored in normal saline at $4^{\circ} \mathrm{C}$. Teeth were decoronated to standardize root canal length at $15 \pm 1 \mathrm{~mm}$. Instrumentation was performed with $14 \mathrm{~mm}$ working length using a crown-down technique with Rotary file Revo-S/Sc2 0.04/25\# (Coltene MicroMega, Besancon, France). All canals were irrigated with $10 \mathrm{~mL}$ of a sodium hypochlorite solution $5.25 \%(\mathrm{NaOCl})$ alternatively between files. After the shaping steps, each canal was irrigated using the same final irrigation protocol based on $2.5 \mathrm{~mL}$ of physiological saline over $90 \pm 5 \mathrm{~s}, 5 \mathrm{~mL}$ of $17 \%$ EDTA solution over $120 \pm 10 \mathrm{~s}, 2.5 \mathrm{~mL}$ of physiological saline over $90 \pm 5$ $\mathrm{s}, 5 \mathrm{~mL}$ of a $6 \% \mathrm{NaOCl}$ over $120 \pm 10 \mathrm{~s}$ followed by a final rinse with $2.5 \mathrm{~mL}$ of physiological saline over $90 \pm 5 \mathrm{~s}$, the canals were then dried with sterile paper points [18].

\section{Obturation Procedure}

The specimens were randomly divided in to 2 groups of 25 samples each.

Group 1: with GP, using BioRoot (Septodont, Saint-Maur-desFosses, France) with single cone technique.

Group 2: with GP, using AH Plus sealer (Dentsplysirona, De Trey Konstanz, Germany) with single cone technique.

After obturation by single cone technique, the coronal opening was sealed with a restorative glass ionomer cement kavitan plus (Spofadental, Jicin, Czech). The samples were then stored in a humid atmosphere (Incubator) at $37^{\circ} \mathrm{C}$ for $72 \mathrm{~h}$.

\section{Microleakage Evaluation}

After the obturation, 15 teeth of each group were prepared to the microleakage evaluation. The surfaces of the samples were dried and two layers of colored nail varnish was applied on the surface $1 \mathrm{~mm}$ short of the apex (Fig. 1a). Roots were then suspended in freshly prepared $2 \%$ methylene blue for 24 hours. The roots were then rinsed for 15 minutes under running water and the varnish layers were sweep off and the teeth were dried. Then the teeth were immersed in a series of solutions using clearing technique in which the teeth become transparent after a process of demineralization, dehydration and immersion in methyl salicylate, provides a three dimensional view of the internal anatomy of root canals without any loss of dental substance, making it easier to view the leakage area (Fig. 1b).

The depth of dye penetration was examined under stereomicroscope at $\times 20$ magnification and microleakage associated with different root canal sealers was evaluated from the end of the root canal to the farthest point. Leakage sign was observed using a digital piacolis a vernier caliper equipment which allows a measurement of $0.01 \mathrm{~mm}$ (Fig. 2).

\section{Optical Numeric Microscope Keyence}

After the obturation procedure, ten teeth of each group were prepared to evaluate the void percentages at $2 \mathrm{~mm}$ from the apex. One section was made perpendicular to the longitudinal axis of

Figure 1. A. Samples after applying two layers of colored nail varnish, B. Samples after using clearing technique.

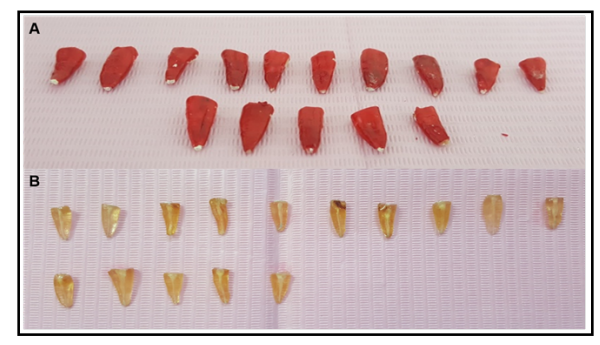


the root tooth at $2 \mathrm{~mm}$ from the apex with a wire saw (Walter EBNER, Le Locle, Switzerland). Subsequently, 1200, 2400, and $4000 \mathrm{SiC}$ abrasive papers were used, under continuous water irrigation, to polish the surface of the specimens. They were first observed using an optical numeric microscope (KEYENCE, Osaka, Japon) and then analyzed using the VHX-5000 communication software (KEYENCE, Osaka, Japon) to measure the total area of the voids at $2 \mathrm{~mm}$ from the apex.

\section{Scanning Electron Microscope (SEM)}

After evaluating the void percentages, all samples were etched with $37 \%$ phosphoric acid (5 s) and $2.5 \% \mathrm{NaOCl}(3 \mathrm{~min})$ and thoroughly rinsed with distilled water for $30 \mathrm{~s}$ in order to eliminate the smear layer resulting from the polishing process and to visualize the sealer infiltrations in dentinal tubules (tags). The samples were sputter-coated with gold-palladium alloys (20/80) using a HUMMER JR sputtering device (Technics, CA, USA). Later on, a Quanta 250 FEG scanning electron microscope SEM (FEI Company, Eindhoven, The Netherlands) functioning with an accelerating voltage of the electrons of $10 \mathrm{kV}$ was used for the observation of the coated specimens.

\section{Statistical Analysis}

The collected data were analyzed with SigmaPlot release 11.2 (Systat Software, Inc., San Jose, CA, USA). Shapiro-Wilk test was used to verify the normality of data. However, when the normality was not verified thus, Kruskal-Wallis test (one-way analysis of variance on ranks) was used to compare the apical micro leakage and void percentages results of AHPlus and Bioroot sealers. A significance level at $\alpha=0.05$ was adopted.

\section{Results}

\section{Microleakage Evaluation}

The study was performed to evaluate the apical sealing ability between BioRoot and AH Plus. The mean values of apical microleakage for the two sealers were recorded (Table 1). Concerning the statistical analysis, it was found that the mean value of dye penetration of the AHPlus group is $(0.76 \pm 0.66) \mathrm{mm}$, while the mean value of BioRoot group $(0.26 \pm 0.06) \mathrm{mm}$ (Table 1$)$. Statistical analysis revealed that the mean dye penetration value for BioRoot was significantly lower than that for AH Plus $(\mathrm{P}<0.05)$.

\section{Keyence and SEM Observations}

No significant statistical difference $(p>0.05)$ was seen in the apical third at $2 \mathrm{~mm}$ from the apex between the Bioroot and AH Plus (Table 1). The void percentages were calculated by dividing the area of the voids measured during the analysis with the total area of the root canal of each specimen (Fig. 3C-E).

Figure 2. (A, B). micro leakage of methylene blue in the apical area (AH plus sealer) examined under stereomicroscope at 20X magnification, $(C, D)$. micro leakage of methylene blue in the apical area BioRoot sealer) examined under stereomicroscope at $20 \mathrm{X}$ magnification.

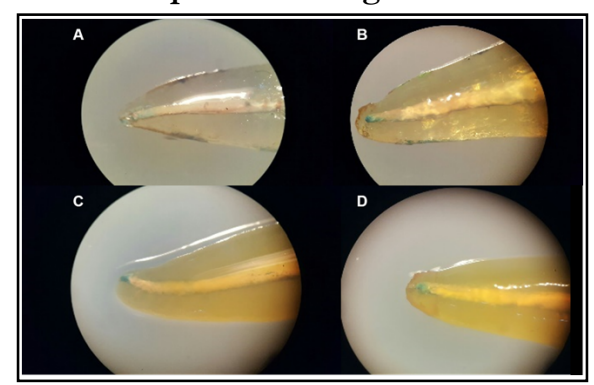

Figure 3. Representative photos of a numeric optical microscope of sectioned root surfaces at $2 \mathrm{~mm}$ from the apex. A. Bioroot; B. AH Plus.

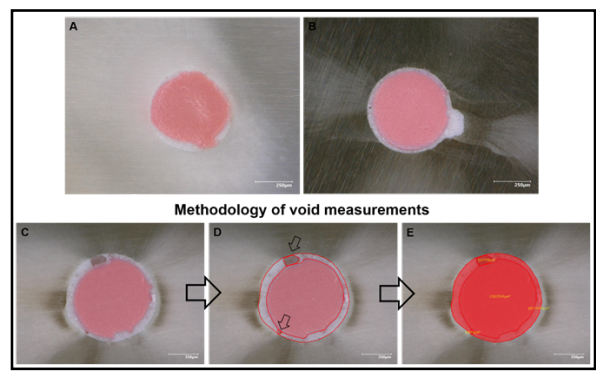

Figure 4. SEM micrograph of the interface between A. AH Plus sealer or B. BioRoot sealer with dentin canal walls showed no sealer infiltrations into dentinal tubules.

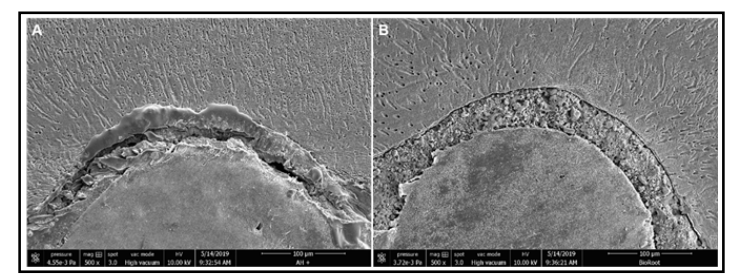


Table 1. Means and standard deviations of dye penetration $(\mathrm{mm})$ and void percentages for the tested materials.

\begin{tabular}{|c|c|c|c|c|}
\hline Test & Sealers used & $\mathbf{N}$ & Mean $(\mathbf{m m})$ & Statistical analysis \\
\hline \multirow{2}{*}{ Dye penetration $(\mathrm{mm})$} & BioRoot & 15 & $0.26 \pm 0.06$ & \multirow{2}{*}{$\mathrm{P}<0.05$} \\
\cline { 2 - 4 } & AH Plus & 15 & $0.76 \pm 0.18$ & \\
\hline \multirow{2}{*}{ Void $(\%)$} & BioRoot & 10 & $3.2 \pm 1.4$ & \multirow{2}{*}{$\mathrm{P}>0.05$} \\
\cline { 2 - 4 } & AH Plus & 10 & $2.9 \pm 1.8$ & \\
\hline
\end{tabular}

SEM analyses assessed the presence or the absence of intra-tubule tags for the two filling techniques analyzed. There were any tag observed at $2 \mathrm{~mm}$ from the apex in all the samples (Fig. 4).

Concerning AH Plus specimens, when observing the areas of gutta-percha, sealer and interface sealer / dentin, it became qualitatively obvious that $\mathrm{AH}$ Plus obturation material, had more void spaces between the sealer material and the dentinal walls (Fig 4a).

\section{Discussion}

The combination between the sealer and the gutta-percha is necessary to ensure a perfect sealing in root canal system, and the sealer paste provide a tough seal at all the entrance along the canal in the distance between the internal walls and the cones and even between the cones themselves $[1,6]$.

Normally, a root canal filling is associated with a hard core, like gutta-percha, and a sealer to better adapt the root canal filling material and complete the seal of the root canal filling in the most effectual manner [19]. Many sealers were produced like oxide zinc base sealer, epoxy resin based sealer, and calcium hydroxide based sealer, but recently calcium silicate based sealer appears like BioRoot and Endosequence BC. The single cone technique was used in this research to obturate the root canals in all samples, Nowadays, there is increasing demand for prompt, simple and efficient obturation technique, which improves practice and causes less stress for patients and clinicians. With the widespread use of rotary NiTi instruments and matched-taper Gutta-percha cones, the single-cone obturation technique has become popular $[20,21]$.

This study which evaluated the sealing ability of BioRoot and AH Plus, concluded that the Leakage cannot be totally eliminated from the fate of a root canal treated teeth; lateral canals, accessory canals, other anatomical variation and periapical pressure play an important role in sealing ability.

The results of this study found that the apical sealing ability of BioRoot sealer exceeded the apical sealing ability of AH Plus sealer, and disagreed with the results of Viapiana et al [22], which found that there is no difference between the sealing ability of the sealers (using techniques fluid transport and leakage of fluorescent microspheres).

The variance between the results of our study and the results of Viapiana et al [21] study, can be attributed to the difference in the preparation and obturation methods of the root canals and also in the apical sealing evaluation methods, while Viapiana et al was adopted the cold lateral condensation technique with a taper 0.06 for preparation, this study used the single cone technique with a taper 0.04 for preparation.
In the present study, we evaluated apical third sections because of the importance of this area in curved canals. Apical leakage was observed in all specimens. Statistical analysis revealed that the lowest apicalleakage was in Group 1 (BioRoot) followed by Group 2 (AH Plus). Group 2 showed a mean value apical leakage of $(0.76 \pm 0.66) \mathrm{mm}$, whereas Group 1 showed a mean value apicalleakage of $(0.26 \pm 0.06) \mathrm{mm}$. The difference between the mean values dye penetration between Groups 1 and 2 was statistically significant $(\mathrm{P}<0.05)$. According to our results, Paranthaman et al [23] showed that BioRoot possessed greater sealing ability compared with the AH Plus at 1 day, 1, 2 and 4 weeks. None of the sealers provided complete sealing at all time periods but BioRoot showed superior sealing ability followed by AH Plus [23]. The null hypothesis of the present study was rejected. The results of microleakage of our study were also confirmed by our SEM observations. Several studies have used SEM to evaluate the dentinal tubule penetration of sealers $[24,25]$. This form of microscopy can be used to observe the surface appearance of sealer in dentinal tubules at high magnification and to determine the density of sealer tags [24]. According to SEM, BioRoot root canal sealers (G1) showed sufficient adaptation to dentin at $1 \mathrm{~mm}$ from the apex (Figure 3b). SEM Micrographs showed lot of voids in the interfaces dentin-sealer in group 2 (Figure 3a). The gaps that have been seen between the AH Plus and dentin walls at $1 \mathrm{~mm}$ from the apex in SEM micrographs validate the microleakage results (Table 1). This finding for the void of the AH Plus is in disagreement with the result of Viapiana et al [26] who used three exvivo evaluation methods. First method was the Micro CT analysis showed that the BioRoot exhibited significantly more percentage of voids than AH Plus. Their other techniques (fluid transport and fluorescent markers) did not show a difference between the sealing ability of the sealers. Huanget al [27] who used SEM analysesfound no significant difference between $\mathrm{AH}$ Plus and an other bioceramic sealer "EndoSequence BC".

SEM analyses revealed that both root canal sealers showed any inter-tubular tag at $1 \mathrm{~mm}$ from the apex (Figure 2). Rouhani et al [28] noted the presence of intertubular dentin tags for AH Plus sealer at 4 and $6 \mathrm{~mm}$ from the apex but in 7 from 14 samples, there weren't any tag penetration. Our SEM observations did not reveal the presence of tags for the other groups. In addition, the number and size of dentinal tubules and dentinal structure (tubule density) in the apical third as well as the obturation technique employed, may affect sealer adaptation and penetration [29]. Mancino et al [30] demonstrated that the use of AH Plus or Bioroot by single cone technique could not penetrate in to the dentinal tubules. It is important to highlight that the presence of sealer tags in to dentinal tubules, could behave as a physical barrier able to entomb bacteria and preventing apical leakage.

This study has limitations. The disadvantage of horizontal sectioning is that only one of section per specimen can be evalu- 
ated. However, the inability to obtain a detailed overall view at low magnification is the main disadvantage of the SEM technique.

\section{Conclusion}

Based on the present study, there is no sealer that completely prevents apical micro leakage. The use of single cone obturation technique with BioRoot sealer showed a high apical sealing ability, and superior than AHPlus sealer. It is known that microorganisms may remain present in the root canal system after instrumentation, so obtaining a strong apical seal is required to prevent bacteria from invading the periodontal apex. The sealing ability of BioRoot by its special characteristic have an important role in the treatment of apical lesion and block of the lateral canals, these points not any sealer can secure it.

\section{References}

[1]. Mancino D, Kharouf N, Hemmerlé J, Haïkel Y. Microscopic and Chemical Assessments of the Filling Ability in Oval-Shaped Root Canals Using Two Different Carrier-Based Filling Techniques. Eur J Dent. 2019 May;13(2):166-171.PubmedPMID: 31574539.

[2]. Zhang W, Li Z, Peng B. Ex vivo cytotoxicity of a new calcium silicate-based canal filling material. Int Endod J. 2010 Sep;43(9):769-74. Pubmed PMID: 20546044.

[3]. Borges ÁH, Orçati Dorileo MC, Dalla Villa R, Borba AM, Semenoff TA, Guedes OA, et al. Physicochemical properties and surfaces morphologies evaluation of MTA FillApex and AH plus. ScientificWorldJournal. 2014;2014:589732. Pubmed PMID: 24883413.

[4]. Lee JK, Kwak SW, Ha JH, Lee W, Kim HC. Physicochemical Properties of Epoxy Resin-Based and Bioceramic-Based Root Canal Sealers. Bioinorg Chem Appl. 2017;2017:2582849. Pubmed PMID: 28210204.

[5]. Ruddle C.Gauging the Terminus: A Novel Method. Endodontic Practice. 2012.

[6]. Kharouf N, Arntz Y, Eid A, Zghal J, Sauro S, Haikel Y, et al . Physicochemical and Antibacterial Properties of Novel, Premixed Calcium Silicate-Based Sealer Compared to Powder-Liquid Bioceramic Sealer. J Clin Med. 2020 Sep 25;9(10):3096. Pubmed PMID: 32992852.

[7]. Siboni F, Taddei P, Prati C, Gandolfi MG. Properties of NeoMTA Plus and MTA Plus cements for endodontics. Int Endod J. 2017 Dec;50 Suppl 2:e83e94. Pubmed PMID: 28452115.

[8]. Kharouf N, Hemmerlé J, Haikel Y, Mancino D. Technical Quality of Root Canal Filling in Preclinical Training at Strasbourg University Using Two Teaching Protocols. Eur J Dent. 2019 Oct;13(4):521-526.Pubmed PMID:31891969.

[9]. Camps J, Jeanneau C, El Ayachi I, Laurent P, About I. Bioactivity of a Calcium Silicate-based Endodontic Cement (BioRoot RCS): Interactions with Human Periodontal Ligament Cells In Vitro. J Endod. 2015 Sep;41(9):1469-73. Pubmed PMID: 26001857.

[10]. Dimitrova-Nakov S, Uzunoglu E, Ardila-Osorio H, Baudry A, Richard G, Kellermann $\mathrm{O}$, et al. In vitro bioactivity of Bioroot ${ }^{\mathrm{TM}}$ RCS, via A4 mouse pulpal stem cells. Dent Mater. 2015 Nov;31(11):1290-7. Pubmed PMID: 26364144

[11]. Arias-Moliz MT, Camilleri J. The effect of the final irrigant on the antimicrobial activity of root canal sealers. J Dent. 2016 Sep;52:30-6. Pubmed PMID: 27377571.

[12]. Patil P, Rathore VP, Hotkar C, Savgave SS, Raghavendra K, Ingale P. A comparison of apical sealing ability between GuttaFlow and $\mathrm{AH}$ plus: An in vitro study. J Int Soc Prev Community Dent. 2016 Jul-Aug;6(4):377-82. Pubmed PMID: 27583228

[13]. Holland R, Murata SS, Dezan E, Garlipp O. Apical leakage after root canal filling with an experimental calcium hydroxide gutta-percha point. J Endod. 1996 Feb;22(2):71-3. Pubmed PMID: 8935021.

[14]. Roggendorf MJ, Ebert J, Petschelt A, Frankenberger R. Influence of moisture on the apical seal of root canal fillings with five different types of sealer. J Endod. 2007 Jan;33(1):31-3. Pubmed PMID: 17185125.

[15]. Brandao CG, De Moraes IG, Bramante CM. Apical sealing ability of ionomeric endodontic sealers. Rev FOB. 2001;9(2):29-34.

[16]. Hammad M, Qualtrough A, Silikas N. Evaluation of root canal obturation: a three-dimensional in vitro study. J Endod. 2009 Apr;35(4):541-4. Pubmed PMID: 19345801.

[17]. Sevimay S, Kalayci A. Evaluation of apical sealing ability and adaptation to dentine of two resin-based sealers. J Oral Rehabil. 2005 Feb;32(2):105-10. Pubmed PMID: 15641975.

[18]. Kharouf N, Pedullà E, La Rosa GRM, Bukiet F, Sauro S, Haikel Y, et al. In Vitro Evaluation of Different Irrigation Protocols on Intracanal Smear Layer Removal in Teeth with or without Pre-Endodontic Proximal Wall Restoration. J Clin Med. 2020 Oct 16;9(10):3325. Pubmed PMID: 33081209.

[19]. Evans JT, Simon JH. Evaluation of the apical seal produced by injected thermoplasticized Gutta-percha in the absence of smear layer and root canal sealer. J Endod. 1986 Mar;12(3):100-7. Pubmed PMID: 3517216.

[20]. Gordon MP, Love RM, Chandler NP. An evaluation of .06 tapered guttapercha cones for filling of .06 taper prepared curved root canals. Int Endod J. 2005 Feb;38(2):87-96. Pubmed PMID: 15667630.

[21]. El Sayed MA, Taleb AA, Balbahaith MS. Sealing ability of three single-cone obturation systems: An in-vitro glucose leakage study. J Conserv Dent. 2013 Nov;16(6):489-93. Pubmed PMID: 24347879.

[22]. Viapiana R, Moinzadeh AT, Camilleri L, Wesselink PR, Tanomaru Filho M, Camilleri J. Porosity and sealing ability of root fillings with gutta-percha and BioRoot RCS or AH Plus sealers. Evaluation by three ex vivo methods. Int Endod J. 2016 Aug;49(8):774-82. Pubmed PMID: 26199130.

[23]. Paranthaman H,Theetharappan P.Evaluation of Sealing Ability of Three Different Sealers, Bioroot RCS, Gutta flow II, AHPlus, An in-Vitro Study. International Journal of Science and Research.2019; 8(2):168-790.

[24]. White RR, Goldman M, Lin PS. The influence of the smeared layer upon dentinal tubule penetration by plastic filling materials. J Endod. 1984 Dec;10(12):558-62. Pubmed PMID: 6440943.

[25]. Vassiliadis LP, Sklavounos SA, Stavrianos CK. Depth of penetration and appearance of Grossman sealer in the dentinal tubules: an in vivo study. J Endod. 1994 Aug;20(8):373-6. Pubmed PMID: 7996102.

[26]. Viapiana R, Guerreiro-Tanomaru J, Tanomaru-Filho M, Camilleri J. Interface of dentine to root canal sealers. J Dent. 2014 Mar;42(3):336-50. Pubmed PMID: 24287256.

[27]. Huang Y, Orhan K, Celikten B, Orhan AI, Tufenkci P, Sevimay S. Evaluation of the sealing ability of different root canal sealers: a combined SEM and micro-CT study. J Appl Oral Sci. 2018 Jan 15;26:e20160584. PubmedPMID: 29340481.

[28]. Rouhani A, Ghoddusi J, Naghavi N, Al-Lawati G. Scanning electron microscopic evaluation of dentinal tubule penetration of Epiphany in severely curved root canals. Eur J Dent. 2013 Oct;7(4):423-428. Pubmed PMID: 24932116.

[29]. Moradi S, Ghoddusi J, Forghani M. Evaluation of dentinal tubule penetration after the use of dentin bonding agent as a root canal sealer. J Endod. 2009 Nov;35(11):1563-6. Pubmed PMID: 19840648.

[30]. Mancino D, Kharouf N, Cabiddu M, Bukiet F, Haïkel Y. Microscopic and chemical evaluation of the filling quality of five obturation techniques in oval-shaped root canals. Clinical oral investigations. 2020 Nov 26:1-9. 